\title{
ON $(1,2)^{*}-\pi g b-C L O S E D$ SETS
}

\author{
D.Sreeja \\ Assistant Professor, \\ Dept. Of Mathematics,CMSCollege Of Science \\ And Commerce,Coimbatore-6.
}

\author{
C.Janaki \\ Assistant Professor, \\ Dept. Of Mathematics,L.R.G Govt.Arts College For \\ Women,Tirupur-4.
}

\begin{abstract}
In this paper we introduce a new class of sets called $(1,2)^{*}$ $\pi \mathrm{gb}$-closed sets and a new class of generalized functions called $(1,2)^{*}-\pi \mathrm{gb}$ - continuous maps and $(1,2)^{*}-\pi \mathrm{gb}$ - irresolute maps in bitopological spaces. Also we obtain basic properties of $(1,2)^{*}-\pi \mathrm{gb}$-closed sets. Further, we introduce a new space called $(1,2)^{*}-\pi \mathrm{gb}$-space.
\end{abstract}

2000 Mathematics Subject Classification: 54E55

\section{Key words and phrases}

$(1,2)^{*}$ - $\pi$ gb-closed, $(1,2)^{*}-\pi$ gb-open, $(1,2)^{*}-\pi$ gb- continuous maps, $(1,2)^{*}-\pi \mathrm{gb}$ - irresolute maps, $(1,2)^{*}-\pi \mathrm{gb}$-space, $(1,2)^{*}$ $\pi \mathrm{gb}-\mathrm{T}_{1 / 2}$ space.

\section{INTRODUCTION}

Andrijevic [3] introduced an innovative class of generalized open sets in a topological space, the so-called bopen sets. Ekici and Caldas [9] already discussed this type of sets under the name of $\gamma$-open sets. The class of b-open sets is contained in the class of semi-pre-open sets and contains all semi-open sets and pre-open sets.It is noteworthy to decipher that there is a unique feature in the class of b-open sets. In subsequent to the findings of these concepts, varied research papers (see $[1,3,6,9,10,19,20$, and 21]) unleashed new results in various respects. Levine [13] introduced the notion of generalized closed sets in topological space and a class of topological spaces called $\mathrm{T}_{1 / 2}$ spaces. In recent years, research on a large scale was carried out in generalizing closedness as the notions of a generalized closed, generalized semi-closed, $\alpha$-generalized closed, generalized semi-pre-open closed sets $[2,7,13,16,17]$. Thivagar et al [22] have introduced the concepts of $(1,2)^{*}$-semi open sets, $(1,2)^{*}$ - $\alpha$-open sets and $(1$, $2)^{*}$ - semi-generalized closed sets, and $(1,2)^{*}-\alpha$-generalized closed sets.

This paper attempts to highlight a new type of generalized sets called $(1,2)^{*}-\pi$ gb-closed sets and a new class of generalized functions called $(1,2)^{*}-\pi \mathrm{gb}$ - continuous maps and $(1,2)^{*}-\pi \mathrm{gb}-$ irresolute maps. These findings results in procuring several characterizations of this class. It analyses its bitopological properties. The application of this set leads to the introduction of a new space called $(1,2)^{*}-\pi \mathrm{gb}$-space.

\section{PRELIMINARIES}

Throughout the present paper $\left(\mathrm{X}, \tau_{1}, \tau_{2}\right),\left(\mathrm{Y}, \sigma_{1}, \sigma_{2}\right)$ and $(\mathrm{Z}$, $\eta 1, \eta 2$ ) (or simply $X, Y, Z$ ) denote bitopological spaces.

Definition 2.1: A subset $A$ of a space $(X, \tau)$ is called

(1) a pre-open set [15] if $A \subset \operatorname{int}(\mathrm{cl}(\mathrm{A}))$ and a pre-closed set if $\operatorname{cl}($ int $(\mathrm{A})) \subset \mathrm{A}$;
(2) a semi-open set[12] if $\mathrm{A} \subset \operatorname{cl}(\operatorname{int}(\mathrm{A}))$ and a semi-closed set if int $(\mathrm{cl}(\mathrm{A})) \subset \mathrm{A}$;

(3) a $\alpha$-open set[18] if $\mathrm{A} \subset$ int $(\operatorname{cl}(\operatorname{int}(\mathrm{A})))$ and a $\alpha$-closed set if $\mathrm{cl}(\operatorname{int}(\mathrm{cl}(\mathrm{A}))) \subset \mathrm{A}$;

(4) a semi-preopen set[1] if $\mathrm{A} \subset \mathrm{cl}(\operatorname{intcl}(\mathrm{A}))$ and a semi-preclosed set if $\operatorname{int}(\mathrm{cl}(\operatorname{int}(\mathrm{A}))) \subset \mathrm{A}$;

(5) a regular open set if $\mathrm{A}=\mathrm{int}(\mathrm{cl}(\mathrm{A}))$ and a regular closed set if $\mathrm{A}=\mathrm{cl}(\mathrm{int}(\mathrm{A})$ );

(6) b-open [3] or sp-open [8], $\gamma$-open [9] if A $\subset$ $\operatorname{cl}(\operatorname{int}(\mathrm{A})) \cup \operatorname{int}(\mathrm{cl}(\mathrm{A}))$.

(7) $\pi \mathrm{g} \alpha$-closed [11] if $\alpha \mathrm{Cl}(\mathrm{A}) \subset \mathrm{U}$ whenever $\mathrm{A} \subset \mathrm{U}$ and $\mathrm{U}$ is $\pi$ open in X.

(8) $\pi$ gb-closed [24] if $\mathrm{bCl}(\mathrm{A}) \subset \mathrm{U}$ whenever $\mathrm{A} \subset \mathrm{U}$ and $\mathrm{U}$ is $\pi$ open in X.

The complement of a b-open set is said to be b-closed [3]. The intersection of all b-closed sets of $\mathrm{X}$ containing $\mathrm{A}$ is called the $\mathrm{b}$-closure of $\mathrm{A}$ and is denoted by $\mathrm{bCl}(\mathrm{A})$. The union of all $\mathrm{b}$ open sets of $\mathrm{X}$ contained in $\mathrm{A}$ is called $\mathrm{b}$-interior of $\mathrm{A}$ and is denoted by bInt(A).

Definition 2.2: A function $\mathrm{f}:(\mathrm{X}, \tau) \rightarrow(\mathrm{Y}, \sigma)$ is called

1) $\pi$ - irresolute: [4] if $\mathrm{f}^{-1}(\mathrm{~V})$ is $\pi$ - closed in $(\mathrm{X}, \tau)$ for every $\pi$ closed of $(Y, \sigma)$.

2) b-irresolute: [9] if for each b-open set $V$ in $Y, f^{-1}(V)$ is bopen in $\mathrm{X}$

3) b-continuous: [9] if for each open set $\mathrm{V}$ in $\mathrm{Y}, \mathrm{f}^{-1}(\mathrm{~V})$ is bopen in $\mathrm{X}$

Definition 2.3: [22] A subset $A$ of $X$ is called $\tau_{1} \tau_{2}$ _open if $S \in \tau_{1} \cup \tau_{2}$ and the complement of $\tau_{1} \tau_{2}$.open set is $\tau_{1} \tau_{2}$. closed.

Definition 2.4: [22] Let A be a subset of X

(i) The $\tau_{1} \tau_{2}$-closure of $\mathrm{A}$, denoted by $\tau_{1} \tau_{2}-\operatorname{cl}(\mathrm{S})$ is defined by $\cap\left\{\mathrm{F} / \mathrm{S} \subset \mathrm{F}\right.$ and $\mathrm{F}$ is $\tau_{1} \tau$-closed $\}$ 
(ii) The $\tau_{1} \tau_{2}$-interior of $\mathrm{A}$, denoted by $\tau_{1} \tau_{2}-\operatorname{int}(\mathrm{S})$ is defined by $\cup\left\{\mathrm{F} / \mathrm{F} \subset \mathrm{S}\right.$ and $\mathrm{F}$ is $\tau_{1} \tau$-open $\}$

\section{Remark 2.5: [23]}

(i) $\tau_{1} \tau_{2}$ int(S) is $\tau_{1,2}$-open for each $\mathrm{S} \subset \mathrm{X}$ and $\tau_{1} \tau_{2}-\mathrm{cl}(\mathrm{S})$ is $\tau_{1,2}$-closed for each $\mathrm{S} \subset \mathrm{X}$.

(ii)A subset $\mathrm{S} \subset \mathrm{X}$ is $\tau_{1,2^{-}}$open iff $\mathrm{S}=\tau_{1} \tau_{2}-\operatorname{int}(\mathrm{S})$ and $\tau_{1,2^{-}}$ closed iff $S=\tau_{1} \tau_{2}-\operatorname{cl}(S)$

(iii) $\tau_{1} \tau_{2}-\operatorname{int}(S)=\operatorname{int}_{\tau 1}(S) \cup$ int $_{\tau 2}(S)$ and $\tau_{1} \tau_{2}-\operatorname{cl}(S)=c l$

${ }_{\tau 1}(\mathrm{~S}) \cup \mathrm{cl}_{\tau 2}(\mathrm{~S})$ for any $\mathrm{S} \subset \mathrm{X}$.

(iv) for any family $\left\{S_{i} / i \in I\right\}$ of subsets of $X$ we have
(a) $\bigcup_{i} \tau_{1} \tau_{2}-\operatorname{int}\left(\mathrm{S}_{\mathrm{i}}\right) \subset \tau_{1} \tau_{2}-\operatorname{int}\left(\bigcup_{i} \mathrm{~S}_{\mathrm{i}}\right)$
(b) $\cup_{i} \tau_{1} \tau_{2}-\operatorname{cl}\left(\mathrm{S}_{\mathrm{i}}\right) \subset \tau_{1} \tau_{2}-\operatorname{cl}\left(\cup_{i} \mathrm{~S}_{\mathrm{i}}\right)$
(c) $\tau_{1} \tau_{2}-\operatorname{int}\left(\bigcap_{i} S_{\mathrm{i}}\right) \subset \bigcap_{i} \tau_{1} \tau_{2}-\operatorname{int}\left(\mathrm{S}_{\mathrm{i}}\right)$
(d) $\quad \tau_{1} \tau_{2}-\operatorname{cl}\left(\bigcap_{i} S_{\mathrm{i}}\right) \subset \bigcap_{i} \tau_{1} \tau_{2}-\operatorname{cl}\left(\mathrm{S}_{\mathrm{i}}\right)$

(v) $\tau_{1,2}$-open sets need not form a topology.

Definition 2.6: The finite union of $\left(1,2^{*}\right.$-regular open sets [5] is said to be $\tau_{1,2}-\pi$ - open. The complement of $\tau_{1,2}-\pi$ open is said to be $\tau_{1,2}-\pi$ - closed.

Definition 2.7: A subset A of a bitopological space (X, $\tau 1, \tau 2)$ is called

(i) $\quad(1,2)^{*}$ - semi-open $\operatorname{set}[22]$ if $\mathrm{A} \subset \tau_{1} \tau_{2-} \operatorname{cl}\left(\tau_{1} \tau_{2}-\right.$ $\operatorname{int}(\mathrm{A}))$

(ii) $\quad(1,2)^{*}$-preopen set [22] if $\mathrm{A} \subset \tau_{1} \tau_{2}$-int $\left(\tau_{1} \tau_{2}\right.$ cl (A))

(iii) $\quad(1,2)^{*}-\alpha-$ open set[22] if $A \subset \tau_{1} \tau_{2}-$ int $\left(\tau_{1} \tau_{2}-\right.$ $\operatorname{cl}\left(\tau_{1} \tau_{2}-\right.$ int $\left.\left.(\mathrm{A})\right)\right)$

(iv) $\quad(1,2)^{*}-\pi \mathrm{g} \alpha$-closed [5] if $(1,2)^{*}-\alpha \mathrm{Cl}(\mathrm{A}) \subset \mathrm{U}$ whenever $\mathrm{A} \subset \mathrm{U}$ and $\mathrm{U}$ is $(1,2)^{*}-\pi$-open in $\mathrm{X}$.

(v) $\quad(1,2) *$-regular open [22] if $A=\tau_{1,2}$-int $\left(\tau_{1,2}\right.$ $\operatorname{cl}(\mathrm{A}))$.

Complement of the $(1,2)^{*}$-regular open set is called $(1,2) *$-regular closed set.

(vi) $\quad(1,2)$-b-open [14] if $\mathrm{A} \subset \tau_{1} \tau_{2-} \operatorname{cl}\left(\tau_{1-} \operatorname{int}(\mathrm{A})\right) \cup$ $\tau_{1-}$ int $\left(\tau_{1} \tau_{2-} \operatorname{cl}(\mathrm{A})\right)$. (vii) $\quad(1,2)^{*}$ - generalized closed (briefly $(1,2)^{*}$-gclosed)[22] if $\tau_{1} \tau_{2}-\operatorname{cl}(\mathrm{A}) \subset \mathrm{U}$ whenever $\mathrm{A} \subset$ $\mathrm{U}$ and $\mathrm{U}$ is $\tau_{1,2^{-}}$open in $\mathrm{X}$.

(viii) $\quad(1,2)^{*}{ }_{-} \alpha$-closure [22] (resp $(1,2)^{*}$-semiclosure) of a subset $\mathrm{A}$ of $\mathrm{X}$,denoted by $(1,2)^{*}$ $\alpha \mathrm{Cl}(\mathrm{A}) \quad\left(\right.$ resp. $\left.\quad(1,2){ }^{*}-\mathrm{SCl}(\mathrm{A})\right)$ is defined to be the intersection of all $(1,2)^{*}-\alpha$ closed (resp. $(1,2)^{*}$-semi-closed) sets containing A.

(ix) $\quad(1,2)^{*}-\alpha$-interior [22] (resp $(1,2){ }^{*}$-semiinterior) of a subset $\mathrm{A}$ of $\mathrm{X}$ denoted by $(1,2)^{*}$ $\alpha$-Int(A) (resp. $\left.(1,2)^{*}-\operatorname{sint}(\mathrm{A})\right)$ is defined to be the union of all $(1,2) *_{-} \alpha$-open(resp. $(1,2) *_{\text {- }}$ semi-open) sets containing A.

(x) $\quad(1,2)^{*}$ - semi-generalized closed (briefly (1, 2) ${ }^{*}$-sg-closed)[22 ] if $(1,2) *-\operatorname{sl}(\mathrm{A}) \subset \mathrm{U}$ whenever $\mathrm{A} \subset \mathrm{U}$ and $\mathrm{U}$ is $(1,2)^{*}$ semi-open in $\mathrm{X}$.

(xi) $\quad(1,2)^{*}$-generalized semi closed (briefly $(1,2)^{*}$ gs-closed) [22] if $(1,2) *_{-} \mathrm{SCl}(\mathrm{A}) \quad \subset \quad \mathrm{U}$ whenever $\mathrm{A} \subset \mathrm{U}$ and $\mathrm{U}$ is $(1,2)^{*} \tau_{1,2}$ open in $\mathrm{X}$.

(xii) $\quad(1,2)^{*}-\alpha$-generalized closed (briefly $(1,2)^{*}$ $\alpha$-closed) [22] if $(1,2)^{*}-\alpha \mathrm{Cl}(\mathrm{A})$ $\subset \mathrm{U}$ whenever $\mathrm{A} \subset \mathrm{U}$ and $\mathrm{U}$ is $\tau_{1,2^{-}}$open in $\mathrm{X}$.

(xiii) $\quad(1,2)^{*}$-generalized $\alpha$-closed (briefly $(1,2)^{*}$ $\mathrm{g} \alpha$-closed)[ 22] if $(1,2)^{*}-\alpha \mathrm{Cl}(\mathrm{A}) \subset \mathrm{U}$ whenever $\mathrm{A} \subset \mathrm{U}$ and $\mathrm{U}$ is $\tau_{1,2^{-}} \alpha$ - open in $\mathrm{X}$.

\section{3. $(1,2)^{*}-\pi g b-c l o s e d ~ s e t s$}

Definition 3.1: A subset A of a bitopological space (X, $\tau 1, \tau 2)$ is called $(1,2)^{*}-\pi$ generalized b-closed (briefly $(1,2)^{*}$ $\pi \mathrm{gb}$-closed) if $\tau_{1} \tau_{2}$-bcl(A) $\subset \mathrm{U}$ whenever $\mathrm{A} \subset \mathrm{U}$ and $\mathrm{U}$ is $\tau_{1} \tau_{2}$ $\pi$-open in X.

Definition 3.2: A subset A of a bitopological space (X, $\tau 1, \tau 2)$ is called $(1,2)^{*}$-b-open [14] if $\mathrm{A} \subset \tau_{1} \tau_{2-} \operatorname{cl}\left(\tau_{1} \tau_{2-}\right.$ $\operatorname{int}(\mathrm{A})) \cup \tau_{1} \tau_{2-} \operatorname{int}\left(\tau_{1} \tau_{2-} \operatorname{cl}(\mathrm{A})\right)$.

Complement of $(1,2)^{*}$-b-open is called $(1,2)^{*}$-b-closed.

Definition 3.3: A subset A of a bitopological space (X, $\tau 1, \tau 2)$ is called $(1,2)^{*}$-generalized b-closed (briefly $(1,2)^{*}$ gb-closed) if $\tau_{1} \tau_{2}$-bcl(A) $\subset \mathrm{U}$ whenever $\mathrm{A} \subset \mathrm{U}$ and $\mathrm{U}$ is $\tau_{1} \tau_{2-}$ open in X.

The complement of a $(1,2)^{*}$ - $\pi$ gb-closed set is called $(1,2)^{*}$ $\pi \mathrm{gb}$-open.

Theorem 3.4: Every $(1,2)^{*}-\alpha$-closed set is $(1,2)^{*}-\pi \mathrm{gb}$ closed set.

Proof: Let A be a $(1,2)^{*}-\alpha$-closed set and $\mathrm{U}$ be any $\tau_{1} \tau_{2}-\pi$ open set containing A. Since A is $(1,2)^{*}-\alpha$-closed set, $(1,2)^{*}$ - 
$\alpha \operatorname{cl}(\mathrm{A})=\mathrm{A} \subset \mathrm{U}$.Every $(1,2)^{*}$ - $\alpha$-closed set is $(1,2)^{*}$-b-closed, $(1,2) *-\operatorname{bcl}(\mathrm{A}) \subset \mathrm{U}$.

Theorem 3.5: Every $\tau_{1} \tau_{2}$-closed set is $(1,2)^{*}$ - $\pi$ gb-closed set.

Proof: Let $A \subset U$ whenever $U$ is $\tau_{1} \tau_{2}-\pi$-open. Since $A$ is $\tau_{1} \tau_{2}$ closed, $\tau_{1} \tau_{2}$-cl $(\mathrm{A})=\mathrm{A} \subset \mathrm{U}$. Every $\tau_{1} \tau_{2}$-closed is $(1,2)^{*}$-b-closed. Hence $\tau_{1} \tau_{2}$-closed set is $(1,2)^{*}-\pi$ gb-closed.

Theorem 3.6: Every $(1,2)^{*}$-g-closed set is $(1,2)^{*}-\pi \mathrm{gb}-$ closed set.

Proof: Let $A \subset X$ be $(1,2)^{*}$-g-closed and $A \subset U$ where $U$ is $\tau_{1} \tau_{2}, \pi$-open.This implies $\tau_{1} \tau_{2}$.cl $(\mathrm{A}) \subset \mathrm{U}$ whenever $\mathrm{A} \subset \mathrm{U}$ and $\mathrm{U}$ is $\tau_{1} \tau_{2}$-open in $\mathrm{X}$. Every $(1,2)^{*}$-g-closed is $(1,2)^{*}$-bclosed. Hence $(1,2)^{*}-\operatorname{bcl}(\mathrm{A}) \subset \mathrm{U}$ and $\mathrm{U}$ is $\tau_{1} \tau_{2}-\pi$-open in $\mathrm{X}$. Hence $(1,2)^{*}$-g-closed set is $(1,2)^{*}-\pi$ gb-closed.

Theorem 3.7: Every $(1,2)^{*}$-b-closed set is $(1,2)^{*}-\pi \mathrm{gb}-$ closed set.

Theorem 3.8: Every $(1,2)^{*}-\pi g \alpha$-closed set is $(1,2)^{*}-\pi \mathrm{gb}-$ closed set.

Theorem 3.9: Every $(1,2)^{*}$-pre-closed set is $(1,2)^{*}-\pi \mathrm{gb}-$ closed set.

Theorem 3.10: Every $(1,2)^{*}$-gb-closed set is $(1,2)^{*}$ $\pi \mathrm{gb}$-closed set.

Theorem 3.11: Every $(1,2)^{*}-\pi$ gp-closed set is $(1,2)^{*}-$ $\pi \mathrm{gb}$-closed set.

Converse of the above statements need not be true as shown in the following examples.

Example 3.12: Consider $X=\{a, b, c, d\}, \tau_{1}=\{\Phi,\{a\},\{d\}$, $\{\mathrm{a}, \mathrm{d}\}, \mathrm{X}\}$ and $\left.\tau_{2}=\{\Phi, \mathrm{c}, \mathrm{d}\},\{\mathrm{a}, \mathrm{c}, \mathrm{d}\} \quad, \mathrm{X}\right\}$.Let $\mathrm{A}=\{\mathrm{c}\} \cdot \tau_{1} \tau_{2^{-}}$ opensets $=\{\Phi,\{\mathrm{a}\},\{\mathrm{d}\},\{\mathrm{a}, \mathrm{d}\},\{\mathrm{c}, \mathrm{d}\},\{\mathrm{a}, \mathrm{c}, \mathrm{d}\}, \mathrm{X}\}$;

$\tau_{1} \tau_{2}$-closed sets $=\{\Phi,\{b, c, d\},\{a, b, c\},\{b, c\},\{a, b\},\{b\}, X\}$;

$(1,2)^{*}-\mathrm{bcl}(\mathrm{A})=\{\mathrm{c}\}$. Hence $\mathrm{A}$ is $(1,2)^{*}-\pi \mathrm{gb}$-closed set but not $\tau_{1} \tau_{2}$-closed, $(1,2)^{*}$-g-closed, $(1,2)^{*}$-gb-closed.

Example 3.13:Consider $X \quad=\{\mathrm{a}, \mathrm{b}, \mathrm{c}, \mathrm{d}\}, \quad \tau_{1}=$ $\{\Phi,\{\mathrm{a}\},\{\mathrm{b}\},\{\mathrm{a}, \mathrm{b}\},\{\mathrm{a}, \mathrm{b}, \mathrm{c}\} \mathrm{X}\}$ and $\tau_{2}=\{\Phi,\{\mathrm{a}, \mathrm{b}, \mathrm{d}\} \mathrm{X}\} . \tau_{1} \tau_{2}-$ open set $=\{\Phi,\{a\},\{b\},\{a, b\},\{a, b, c\},\{a, b, d\}, X\} \tau_{1} \tau_{2}$-closed set $=$ $\{\Phi,\{\mathrm{a}, \mathrm{c}, \mathrm{d}\},\{\mathrm{b}, \mathrm{c}, \mathrm{d}\},\{\mathrm{c}, \mathrm{d}\},\{\mathrm{d}\},\{\mathrm{c}\}, \mathrm{X}\}$. Let $\mathrm{A}=\{\mathrm{a}, \mathrm{b}, \mathrm{c}\}$. Here $\mathrm{A}$ is $(1,2)^{*}-\pi$ gb-closed but not $(1,2)^{*}$-b-closed

Example 3.14: Let $\mathrm{X}=\{\mathrm{a}, \mathrm{b}, \mathrm{c}, \mathrm{d}, \mathrm{e}\}$ and $\tau_{1}=$ $\{\Phi,\{\mathrm{a}, \mathrm{b}\}, \mathrm{a}, \mathrm{b}, \mathrm{c}, \mathrm{d}\}, \mathrm{X}\}, \tau_{2}=\{\Phi,\{\mathrm{c}, \mathrm{d}\},\{\mathrm{a}, \mathrm{b}, \mathrm{c}, \mathrm{d}\}, \mathrm{X}\}, \mathrm{A}=\{\mathrm{a}\} . \tau_{1} \tau_{2^{-}}$ open sets $=\{\Phi,\{a, b\},\{c, d\},\{a, b, c, d\}, X\}$;

$\tau_{1} \tau_{2}$-closed sets $=\{\Phi,\{\mathrm{c}, \mathrm{d}, \mathrm{e}\},\{\mathrm{a}, \mathrm{b}, \mathrm{e}\},\{\mathrm{e}\}, \mathrm{X}\}$;

$\tau_{1} \tau_{2}-\pi$-opensets $=\{\Phi,\{\mathrm{a}, \mathrm{b}\},\{\mathrm{c}, \mathrm{d}\},\{\mathrm{a}, \mathrm{b}, \mathrm{c}, \mathrm{d}\}, \mathrm{X}\}$

A is $(1,2)^{*}-\pi$ gb- closed but not, $(1,2)^{*}-\alpha$ - closed, $(1,2)^{*}-\pi g \alpha-$ closed.

Example 3.15: Let $\mathrm{X}=\{\mathrm{a}, \mathrm{b}, \mathrm{c}, \mathrm{d}, \mathrm{e}\}$ and $\tau_{1}=$ $\{\Phi,\{\mathrm{a}, \mathrm{b}\}, \mathrm{a}, \mathrm{b}, \mathrm{c}, \mathrm{d}\}, \mathrm{X}\}, \tau_{2}=\{\Phi,\{\mathrm{c}, \mathrm{d}\},\{\mathrm{a}, \mathrm{b}, \mathrm{c}, \mathrm{d}\}, \mathrm{X}\}, \mathrm{A}=\{\mathrm{a}, \mathrm{b}\}$. $\tau_{1} \tau_{2}$-opensets $=\{\Phi,\{\mathrm{a}, \mathrm{b}\},\{\mathrm{c}, \mathrm{d}\},\{\mathrm{a}, \mathrm{b}, \mathrm{c}, \mathrm{d}\}, \mathrm{X}\}$; $\tau_{1} \tau_{2}$-closed sets $=\{\Phi,\{\mathrm{c}, \mathrm{d}, \mathrm{e}\},\{\mathrm{a}, \mathrm{b}, \mathrm{e}\},\{\mathrm{e}\}, \mathrm{X}\}$

$\tau_{1} \tau_{2}-\pi$-opensets $=\{\Phi,\{\mathrm{a}, \mathrm{b}\},\{\mathrm{c}, \mathrm{d}\},\{\mathrm{a}, \mathrm{b}, \mathrm{c}, \mathrm{d}\}, \mathrm{X}\}$;

A is $(1,2)^{*}-\pi \mathrm{gb}$ - closed but not, $(1,2)^{*}$-pre- closed, $(1,2)^{*}$ $\pi \mathrm{gp}$ - closed.

Remark 3.16: Finite union of $(1,2)^{*}-\pi \mathrm{gb}$ - closed sets need not be $(1,2) *-\pi \mathrm{gb}$-closed.

Example 3.17: Consider $X=\{a, b, c\}, \tau_{1}=\{\Phi,\{a\},\{a, b\}, X\}$ and $\tau_{2}=\{\Phi,\{b\},\{a, b\}, X\} . \tau_{1} \tau_{2}$-open set $=\{\Phi,\{a\},\{b\},\{a, b\}$ $, \mathrm{X}\}, \tau_{1} \tau_{2}$-closed set $=\quad\{\Phi,\{\mathrm{a}, \mathrm{c}\},\{\mathrm{b}, \mathrm{c}\}\{\mathrm{c}\}, \mathrm{X}\} . \quad$ Let $\mathrm{A}=\{\mathrm{a}\}, \mathrm{B}=\{\mathrm{b}\}$. Here $\mathrm{A}$ and $\mathrm{B}$ are $(1,2) *-\pi \mathrm{gb}-$ closed but $\mathrm{A} \cup$ $B=\{a, b\}$ is not $(1,2)^{*}-\pi$ gb-closed since $\{a, b\}$ is $\tau_{1} \tau_{2}-\pi$-open and $b c l(\{a, b\})=X$.

Remark 3.18: Finite intersection of $(1,2)^{*}-\pi \mathrm{gb}$-closed sets need not be $(1,2)^{*}-\pi \mathrm{gb}$ - closed.

Example 3.19:Consider $X \quad=\{\mathrm{a}, \mathrm{b}, \mathrm{c}, \mathrm{d}\}, \quad \tau_{1}=$ $\{\Phi,\{a\},\{b\},\{a, b\},\{a, b, c\} X\}$ and $\tau_{2}=\{\Phi,\{a, b, d\} X\} . \tau_{1} \tau_{2}-$ open set $=\{\Phi,\{a\},\{b\},\{a, b\},\{a, b, c\},\{a, b, d\}, X\} \tau_{1} \tau_{2}$-closed set $=$ $\{\Phi,\{\mathrm{a}, \mathrm{c}, \mathrm{d}\},\{\mathrm{b}, \mathrm{c}, \mathrm{d}\},\{\mathrm{c}, \mathrm{d}\},\{\mathrm{d}\},\{\mathrm{c}\}, X\}$. Let

$\mathrm{A}=\{\mathrm{a}, \mathrm{b}, \mathrm{c}\}, \mathrm{B}=\{\mathrm{a}, \mathrm{b} \cdot \mathrm{d}\}$. Here $\mathrm{A}$ and $\mathrm{B}$ are $(1,2)^{*}-\pi \mathrm{gb}$-closed but $\mathrm{A} \cap \mathrm{B}=\{\mathrm{a}, \mathrm{b}\}$ is not $(1,2)^{*}-\pi \mathrm{gb}$ - closed, since $\{\mathrm{a}, \mathrm{b}\}$ is $\tau_{1} \tau_{2}-\pi$ open and $(1,2)^{*}-\operatorname{bcl}(\{\mathrm{a}, \mathrm{b}\})=\mathrm{X}$.

Theorem 3.20: If a set $A$ is $(1,2)^{*}-\pi \mathrm{gb}$-closed in $\left(X, \tau_{1}\right.$ $\left.\tau_{2}\right)$, then $(1,2)^{*}$.bcl(A) $-\mathrm{A}$ does not contain any non empty $\tau_{1} \tau_{2}-\pi$-closed set.

Proof: Let A be $(1,2)^{*}-\pi$ gb-closed and $\mathrm{F}$ be a non empty $\tau_{1} \tau_{2}-\pi$-closed set such that $\mathrm{F} \subset(1,2)^{*}$ bcl(A)-A. Since A is $(1$, $2)^{*}-\pi$ gb-closed, $\mathrm{A} \subset \mathrm{X}-\mathrm{F}$ where $\mathrm{X}-\mathrm{F}$ is $\tau_{1} \tau_{2}-\pi$-open implies $(1$, $2)^{*}-\mathrm{bcl}(\mathrm{A}) \subset \mathrm{X}-\mathrm{F}$. Hence $\mathrm{F} \subset \mathrm{X}-(1,2)^{*}$. bcl(A).Now $\mathrm{F} \subset(1$, $2)^{*}-\mathrm{bcl}(\mathrm{A}) \cap\left(\mathrm{X}-(1,2)^{*}-\mathrm{bcl}(\mathrm{A})\right)=\Phi$ which is a contradiction. Therefore $(1,2)^{*}-\operatorname{bcl}(\mathrm{A})$ does not contain any non empty $\tau_{1} \tau_{2}$ $-\pi$ - closed set.

Corollary 3.21: Let A be $(1,2)^{*} \pi$ gb -closed in $\left(X, \tau_{1}\right.$, $\left.\tau_{2}\right)$ Then $A$ is $(1,2)^{*}$-b-closed iff $(1,2)^{*}-\operatorname{bcl}(\mathrm{A})-\mathrm{A}$ is $\tau_{1} \tau_{2}-\pi$ closed.

Proof: Let A be $(1,2)^{*}-\mathrm{b}$-closed. Then $(1,2)^{*}-\mathrm{bcl}(\mathrm{A})=\mathrm{A}$. This implies $(1,2)^{*}$-bcl(A)-A= $\Phi$ which is $\tau_{1} \tau_{2}-\pi$ - closed. Assume $(1,2)^{*}-\mathrm{bcl}(\mathrm{A})-\mathrm{A}$ is $\tau_{1} \tau_{2}-\pi$ - closed. Then $(1,2)^{*}$ $\operatorname{bcl}(\mathrm{A})-\mathrm{A}=\Phi$. Hence, $(1,2)^{*}-\mathrm{bcl}(\mathrm{A})=\mathrm{A}$.

Theorem 3.22: If $\mathrm{A}$ is $\tau_{1} \tau_{2}-\pi$-open and $(1,2)^{*}-\pi \mathrm{gb}-$ closed subset of $\left(X, \tau_{1}, \tau_{2}\right)$, then $A$ is $(1,2)^{*}$-b-closed of $(X$, $\left.\tau_{1,} \tau_{2}\right)$

Proof: Since $A \subset A$ and $A$ is $\tau_{1} \tau_{2}-\pi$-open, $(1,2)^{*}-\pi$ gb-closed then $(1,2)^{*}-\operatorname{bcl}(\mathrm{A}) \subset \mathrm{A}$. Hence $\mathrm{A}=(1,2)^{*}-\mathrm{bcl}(\mathrm{A})$. Therefore A is $(1,2) *$-b-closed.

Theorem 3.23: Let A is $(1,2)^{*}-\pi$ gb-closed subset of $(X$, $\left.\tau_{1}, \tau_{2}\right)$ and $B$ is any set such that $A \subset B \subset(1,2)^{*}$-bcl $(A)$, then $\mathrm{B}$ is $(1,2)^{*}-\pi \mathrm{gb}$-closed subset of $\left(\mathrm{X}, \tau_{1}, \tau_{2}\right)$.

Proof: Let $B \subset \mathrm{U}$ and $\mathrm{U}$ be $(1,2)^{*}-\pi$-open of $\left(\mathrm{X}, \tau_{1}, \tau_{2}\right)$. Given A $\subset$ B. Then A $\subset$ U.Since A is $(1,2)^{*}-\pi$ gb- closed, 
$\mathrm{A} \subset \mathrm{U}$ implies $(1,2)^{*}-\mathrm{bcl}(\mathrm{A}) \subset \mathrm{U}$. Also since $\mathrm{B} \subset(1,2)^{*}$-bcl (A) it follows that $(1,2)^{*}$-bcl(B) $\subset(1,2)^{*}$-b cl $\left((1,2)^{*}\right.$-bcl (A)) $=(1,2)^{*}$-bcl (B) $\subset$ U. Hence B is a $(1,2)^{*}-\pi$ gb-closed subset of $\left(\mathrm{X}, \tau_{1}, \tau_{2}\right)$.

Theorem 3.24: Let $\mathrm{B} \subseteq \mathrm{A} \subseteq \mathrm{X}$ where $\mathrm{A}$ is $(1,2)^{*}-\pi \mathrm{gb}$ closed and $\tau_{1} \tau_{2}-\pi$ - open set. Then B is $(1,2)^{*}-\pi \mathrm{gb}$ - closed relative to $\mathrm{A}$ iff $\mathrm{B}$ is $(1,2)^{*}$ - $\pi \mathrm{gb}$-closed in $\mathrm{X}$.

Proof: Let $\mathrm{B} \subseteq \mathrm{A} \subseteq \mathrm{X}$ where $\mathrm{A}$ is $(1,2)^{*}-\pi \mathrm{gb}$ - closed and $\tau_{1} \tau_{2}$ $-\pi$-open set. Let $B$ be $(1,2)^{*}-\pi g b$-closed in $A$. Let $B \subset U$ where $U$ is $\tau_{1} \tau_{2}-\pi$-open in $X$. Since $B \subset A, B=B \cap A \subset U \cap A$, this implies $(1,2)^{*}-\mathrm{bcl}(\mathrm{B})=(1,2)^{*}-\mathrm{bcl}_{\mathrm{A}}(\mathrm{B}) \subset \mathrm{U} \cap \mathrm{A} \subset \mathrm{U}$ .Hence is $(1,2)^{*}-\pi$ gb-closed in $\mathrm{X}$.

Let $\mathrm{B}$ be $(1,2)^{*}-\pi$ gb-closed in $\mathrm{X}$. Let $\mathrm{B} \subset \mathrm{O}$ where $\mathrm{O}$ is $\tau_{1} \tau_{2}$ $\pi$-open in $\mathrm{A}$. Then $\mathrm{O}=\mathrm{U} \cap \mathrm{A}$ where $\mathrm{U}$ is $\tau_{1} \tau_{2}-\pi$-open in $\mathrm{X}$. This implies $\mathrm{B} \subset \mathrm{O}=\mathrm{U} \cap \mathrm{A} \subset \mathrm{U}$. Since $\mathrm{B}$ is $(1,2)^{*}-\pi \mathrm{gb}$-closed in $\mathrm{X}$, $(1, \quad 2)^{*}-\operatorname{bcl}(\mathrm{B}) \subset \mathrm{U}, \quad(1, \quad 2)^{*}-$ bcl $_{\mathrm{y}}(\mathrm{B}) \subset \mathrm{A} \cap \quad(1, \quad 2)^{*}-$ $\mathrm{bcl}(\mathrm{B}) \subset \mathrm{U} \cap \mathrm{A}=\mathrm{O}$ that is $\mathrm{bcl}_{\mathrm{A}}(\mathrm{B}) \subset \mathrm{O}$. Hence, $\mathrm{B}$ is $(1,2)^{*}-\pi \mathrm{gb}-$ closed relative to $\mathrm{A}$.

\section{APPLICATIONS}

Definition 4.1: A subset A of $\left(\mathrm{X}, \tau_{1}, \tau_{2}\right)$ is called $(1,2)^{*}$ $\pi \mathrm{gb}$ - open if and only if its compliment is $(1,2)^{*}-\pi \mathrm{gb}$ - closed in $\left(\mathrm{X}, \tau_{1}, \tau_{2}\right)$.

Remark 4.2: $(1,2)^{*}-\mathrm{bcl}(\mathrm{X}-\mathrm{A})=\mathrm{X}-(1,2)^{*}-\operatorname{bint}(\mathrm{A})$

Theorem 4.3: Every $\tau_{1} \tau_{2}$-open set is $(1,2)^{*}-\pi$ gb-open.

Converse of the above theorem need not be true as shown in the following example.

Example 4.4: Let $X=\{a, b, c\} . \tau_{1}=\{\Phi,\{a, b\}, X\} ; \tau_{2}=\{\Phi$ $,\{\mathrm{a}, \mathrm{c}\}, \mathrm{X}\} \cdot \tau_{1} \tau_{2}-$ open set $=\{\Phi,\{\mathrm{a}, \mathrm{c}\},\{\mathrm{a}, \mathrm{b}\}, \mathrm{X}\} ;\{\mathrm{a}\}$ is $(1,2)^{*_{-}}$ $\pi \mathrm{gb}$-open but not $\tau_{1} \tau_{2}$-open.

Theorem 4.5: A subset $\mathrm{A}$ of $\left(\mathrm{X}, \tau_{1}, \tau_{2}\right)$ is $(1,2)^{*}-\pi \mathrm{gb}-$ open iff $\mathrm{F} \subset(1,2)^{*}$-bint $(\mathrm{A})$ whenever $\mathrm{F}$ is $\tau_{1} \tau_{2}-\pi$-closed and $\mathrm{F} \subset \mathrm{A}$

Proof: Necessity: Let A be $(1,2)^{*}-\pi \mathrm{gb}-$ open in $\left(\mathrm{X}, \tau_{1}\right.$, $\left.\tau_{2}\right)$. Let $F$ be $\tau_{1} \tau_{2}-\pi$-closed and $F \subset A$. Then X-A $\subset$ X-F where $\mathrm{X}-\mathrm{F}$ is $\tau_{1} \tau_{2}-\pi$-open. By assumption $(1,2)^{*}-$ bcl $(\mathrm{X}-\mathrm{A}) \subset$ X-F (by theorem 3.18). By remark 4.2, X- $(1,2)^{*}$-bint (A) $\subset$ $\mathrm{X}$-F. Hence $\mathrm{F} \subset(1,2)^{*}$ - bint $(\mathrm{A})$.

Sufficiency: Suppose $F$ is $\tau_{1} \tau_{2}-\pi$ - closed and $F \subset$ A such that $\mathrm{F} \subset(1,2)^{*}$-bint(A).Let $\mathrm{X}$-A $\subset \mathrm{U}$ where $\mathrm{U}$ is $\tau_{1} \tau_{2}-\pi$-open.

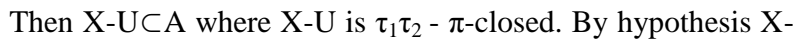
$\mathrm{U} \subset(1,2)^{*}$-bint $(\mathrm{A}) \Rightarrow \mathrm{X}-(1,2)^{*}$-bint $(\mathrm{A}) \subset \mathrm{U} \Rightarrow(1,2)^{*}$-bcl $(\mathrm{X}-$ A) $\subset$ U. Thus X-A is $(1,2)^{*}-\pi \mathrm{gb}$-closed and A is $(1,2)^{*}-\pi \mathrm{gb}-$ open.
Theorem 4.6: If $(1,2)^{*}$ - bint $(\mathrm{A}) \subset \mathrm{B} \subset \mathrm{A}$ and $\mathrm{A}$ is $(1$, $2)^{*}-\pi \mathrm{gb}$-open then B is $(1,2)^{*}-\pi \mathrm{gb}$-open.

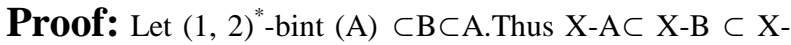
$(1,2)^{*}$-bint A.(ie) X-A $\subset \mathrm{X}-\mathrm{B} \subset(1,2)^{*}$ - bcl $(\mathrm{X}-\mathrm{A})$ by remark 4.2. Since $\mathrm{A}$ is $(1,2)^{*}-\pi \mathrm{gb}-$ open, $\mathrm{X}-\mathrm{A}$ is $(1,2)^{*}-\pi \mathrm{gb}$ - closed. Since X-A is $(1,2)^{*}-\pi$ gb- closed, by theorem 3.23 , (X-A) $\subset$ $(\mathrm{X}-\mathrm{B}) \subset(1,2)^{*}-\mathrm{bcl}(\mathrm{X}-\mathrm{A}) \Rightarrow(\mathrm{X}-\mathrm{B})$ is $(1,2)^{*}-\pi \mathrm{gb}-$ closed. Hence B is $(1,2)^{*}-\pi \mathrm{gb}-$ open.

Definition 4.7: A space $\left(\mathrm{X}, \tau_{1}, \tau_{2}\right)$ is called a $(1,2)^{*}-\pi \mathrm{gb}-$ $\mathrm{T}_{1 / 2}$ space if every $(1,2)^{*}-\pi \mathrm{gb}$ - closed set is b-closed.

Theorem 4.8: For a bitopological space $\left(\mathrm{X}, \tau_{1}, \tau_{2}\right)$ the following are equivalent

(i) $\left(\mathrm{X}, \tau_{1}, \tau_{2}\right)$ is $(1,2)^{*}-\pi \mathrm{gb}-\mathrm{T}_{1 / 2}$ space.

(ii)Every singleton set is $\tau 1 \tau 2$ - $\pi$-closed or $(1,2)^{*}$-b-open.

Proof: To prove (i) implies (ii).Let $\mathrm{X}$ be a $(1,2)^{*}-\pi \mathrm{gb}-\mathrm{T}_{1 / 2}$ space .Let $x \in X$ and assuming that $\{x\}$ is not $\tau_{1} \tau_{2}-\pi$ - closed of $\left(X, \tau_{1}, \tau_{2}\right)$. Then clearly $X-\{X\}$ is not $\tau_{1} \tau_{2}-\pi$ - open. So $X$ is the only $\tau_{1} \tau_{2}-\pi$ - open set containing $X-\{x\}$. Hence $X-\{x\}$ is trivially a $(1,2)^{*}-\pi$ gb-closed set. Since $\left(\mathrm{X}, \tau_{1}, \tau_{2}\right)$ is $(1,2)^{*}$ $\pi \mathrm{gb}-\mathrm{T}_{1 / 2}$ space, $\mathrm{X}-\{\mathrm{x}\}$ is $(1,2)^{*}$ - $\mathrm{b}$-closed set of $\left(\mathrm{X}, \tau_{1}, \tau_{2}\right)$ or equivalently, $\{\mathrm{X}\}$ is $(1,2)^{*}$-b-open set of $\left(\mathrm{X}, \tau_{1}, \tau_{2}\right)$.

(ii) implies (i) Assume every singleton of $\left(\mathrm{X}, \tau_{1}, \tau_{2}\right)$ is either $(1,2)^{*}-\pi$-closed or $(1,2)^{*}$-b-open.Let A be a $(1,2)^{*}-\pi \mathrm{gb}$ closed set of $\left(X, \tau_{1}, \tau_{2}\right)$. Obviously AC $(1,2)^{*}$-bcl(A). Let $\{x\}$ be $(1,2)^{*}$-bcl (A) .To prove $(1,2)^{*}-\operatorname{bcl}(\mathrm{A}) \subset \mathrm{A}$.

Case (i): Let $\{\mathrm{x}\}$ be $\tau_{1} \tau_{2}-\pi$ - closed. Suppose $\{\mathrm{x}\}$ does not belong to $\mathrm{A}$. Then $\{\mathrm{x}\} \in \operatorname{bcl}(\mathrm{A})-\mathrm{A}$, by theorem 3.18, which is a contradiction. Therefore $\{\mathrm{x}\} \in \mathrm{A}$. Hence $(1,2)^{*}$-bcl(A) $\subset \mathrm{A}$.

Case(ii): Let $\{\mathrm{x}\}$ be $(1,2)^{*}$-b-open .Since $\{\mathrm{x}\} \in(1,2)^{*}$ $\mathrm{bcl}(\mathrm{A})$,we have $\{\mathrm{x}\} \cap \mathrm{A} \neq \Phi$,This implies $\{\mathrm{x}\} \in \mathrm{A}$. Therefore $(1,2)^{*}$-bcl (A) $\subset$ A. Hence Therefore $(1,2)^{*}$-bcl $(\mathrm{A})=\mathrm{A}$ or equivalently $\mathrm{A}$ is $(1,2)^{*}$-b-closed. Hence $\left(\mathrm{X}, \tau_{1}, \tau_{2}\right)$ is $(1,2)^{*}$ $\pi \mathrm{gb}-\mathrm{T}_{1 / 2}$ space.

Definition 4.9: A topological space $\mathrm{X}$ is a $(1,2)^{*}-\pi \mathrm{gb}-$ space if every $(1,2)^{*}-\pi \mathrm{gb}$ - closed set is $\tau_{1} \tau_{2}$-closed.

Theorem 4.10: Every $(1,2)^{*}-\pi \mathrm{gb}$-space is $(1,2)^{*}-\pi \mathrm{gb}-$ $\mathrm{T}_{1 / 2}$ space

Proof: Suppose A is $(1,2)^{*}-\pi \mathrm{gb}$-closed in $\left(\mathrm{X}, \tau_{1}, \tau_{2}\right)$. By assumption $\mathrm{A}$ is $\tau_{1} \tau_{2}$-closed. Every $\tau_{1} \tau_{2}$-closed set is $\tau_{1} \tau_{2}$-bclosed. Therefore A is $(1,2)^{*}-\pi \mathrm{gb}-\mathrm{T}_{1 / 2}$ space.

Converse of the above theorem need not be true as shown in the following example.

Example 4.11: Let $\quad \mathrm{X}=\{\mathrm{a}, \mathrm{b}, \mathrm{c}\} ; \quad \tau_{1}=\{\Phi,\{\mathrm{a}\}, \mathrm{X}\}$; $\{\Phi,\{b\}, X\} ; \tau_{1} \tau_{2}$-open sets $=\{\Phi,\{a\},\{b\}, X\} .\left(X, \tau_{1}, \tau_{2}\right)$ is $(1$, $2)^{*}-\pi \mathrm{gb}-\mathrm{T}_{1 / 2}$ space but not $(1,2)^{*}$ - $\pi \mathrm{gb}$-space since $\{\mathrm{b}\}$ is $(1$, $2)^{*}$ - $\pi$ gb-closed but not $\tau_{1} \tau_{2}$-closed. 


\section{5. $(1,2)^{*}-\pi g b-$ continuous and $(1,2)^{*}-\pi g b-$ irresolute functions}

Definition 5.1: A function $\mathrm{f}:\left(\mathrm{X}, \tau_{1}, \tau_{2}\right) \rightarrow\left(\mathrm{Y}, \sigma_{1}, \sigma_{2}\right)$ is called $(1,2)^{*}-\pi \mathrm{gb}$ - continuous if every $\mathrm{f}^{-1}(\mathrm{~V})$ is $(1,2)^{*}(1,2)^{*}$ $-\pi$ gb- closed in $\left(\mathrm{X}, \tau_{1}, \tau_{2}\right)$ for every $\sigma_{1} \sigma_{2}$-closed set $\mathrm{V}$ of $(\mathrm{Y}$, $\left.\sigma_{1}, \sigma_{2}\right)$

Definition 5.2: A function $\mathrm{f}:\left(\mathrm{X}, \tau_{1}, \tau_{2}\right) \rightarrow\left(\mathrm{Y}, \sigma_{1}, \sigma_{2}\right)$ is called $(1,2)^{*}-\pi \mathrm{gb}$ - irresolute if $\mathrm{f}^{-1}(\mathrm{~V})$ is $(1,2)^{*}-\pi \mathrm{gb}$ - closed in $\left(\mathrm{X}, \tau_{1}, \tau_{2}\right)$ for every $(1,2)^{*}-\pi \mathrm{gb}$-closed set $\mathrm{V}$ in $\left(\mathrm{Y}, \sigma_{1}, \sigma_{2}\right)$.

Remark 5.3: A map f: $\left(\mathrm{X}, \tau_{1}, \tau_{2}\right) \rightarrow\left(\mathrm{Y}, \sigma_{1}, \sigma_{2}\right)$ is $(1,2){ }^{*}$ $\pi \mathrm{gb}$ - irresolute iff the inverse image of every $(1,2) *-\pi \mathrm{gb}$-open in $\mathrm{Y}$ is $(1,2)^{*}-\pi \mathrm{gb}$-open in $\mathrm{X}$.

Remark 5.4: Every $(1,2)^{*}-\pi \mathrm{gb}$ - irresolute function is (1, $2)^{*}-\pi$ gb- continuous.

Proof: Let $\mathrm{f}$ be $(1,2)^{*}-\pi \mathrm{gb}$-irresolute. Let $\mathrm{V}$ be a $\tau_{1} \tau_{2}$ closed set in $\left(\mathrm{Y}, \sigma_{1}, \sigma_{2}\right)$.Since every $\tau_{1} \tau_{2}$-closed set is $(1,2)^{*}$ $\pi \mathrm{gb}$-closed, $\mathrm{V}$ is $(1,2)^{*}-\pi \mathrm{gb}$-closed in $\left(\mathrm{Y}, \sigma_{1}, \sigma_{2}\right)$. Since $\mathrm{f}$ is $(1$, $2)^{*}-\pi \mathrm{gb}$ - irresolute, $\mathrm{f}^{-1}(\mathrm{~V})$ is $(1,2)^{*}-\pi \mathrm{gb}$-closed $\mathrm{in}\left(\mathrm{X}, \tau_{1}, \tau_{2}\right)$ Therefore $\mathrm{f}$ is $(1,2) *-\pi \mathrm{gb}$ - continuous.

Converse need not be true as seen in the following example.

Example 5.5: Consider $\mathrm{X}=\mathrm{Y}=\{\mathrm{a}, \mathrm{b}, \mathrm{c}\}, \tau_{1}=\{\Phi,\{\mathrm{a}\},\{\mathrm{c}\}$, $\{a, c\}, X\}, \tau_{2}=\{\Phi,\{a\}, X\}$. So the sets in $\{\Phi,\{a\},\{c\}\{a, c\}, X\}$ are $\tau_{1} \tau_{2}$-open sets in $\{\Phi,\{b, c\}\{a, b\},\{b\} X\}$ are $\tau_{1} \tau_{2}$-closed.Let $\sigma_{1}=\{\Phi,\{\mathrm{a}\}, \mathrm{Y}\}$ and $\sigma_{2}=\{\Phi, \mathrm{Y}\}$. So the sets in $\{\Phi,\{\mathrm{a}\}, \mathrm{Y}\}$ are $\sigma_{1} \sigma_{2}$-open and the sets in $\{\Phi,\{b, c\}, Y\}$ are $\sigma_{1} \sigma_{2}$-closed. Define f: $\left(\mathrm{X}, \tau_{1}, \tau_{2}\right) \rightarrow\left(\mathrm{Y}, \sigma_{1}, \sigma_{2}\right)$ by $\mathrm{f}(\mathrm{a})=\mathrm{a}, \mathrm{f}(\mathrm{b})=\mathrm{b}, \mathrm{f}(\mathrm{c})=\mathrm{c}$. Then $\mathrm{f}$ is $(1,2) *_{-} \pi \mathrm{gb}$ - continuous map but not $(1,2){ }_{-}-\pi \mathrm{gb}-$ irresolute since the inverse image of $(1,2)^{*}-\pi \mathrm{gb}$ - closed sets $\{\mathrm{a}, \mathrm{c}\}$ in $\mathrm{Y}$ is not $(1,2)^{*}-\pi$ gb-closed set in $\mathrm{X}$.

Theorem 5.6: Every $(1,2) *$-continuous map is $(1,2)^{*}$ $\pi \mathrm{gb}-$ continuous map.

Converse of the above theorem need not be true as shown in the following example.

Let $\mathrm{X}=\mathrm{Y}=\{\mathrm{a}, \mathrm{b}, \mathrm{c}\}, \tau_{1}=\{\Phi,\{\mathrm{a}\}, \mathrm{X}\}$ and $\tau_{2}=\{\Phi, \mathrm{X}\}$ So the sets in $\{\Phi,\{a\}, X\}$ are $\tau_{1} \tau_{2}$-open and sets in $\{\Phi,\{b, c\}, X\}$ are $\tau_{1} \tau_{2}-$ closed. Let $\sigma_{1}=\{\Phi,\{a\},\{a, b\}, Y\}$ and $\sigma_{2}=\{\Phi,\{a, b\}, Y\}$. So the sets in $\{\Phi,\{\mathrm{a}\},\{\mathrm{a}, \mathrm{b}\}, \mathrm{Y}\}$ are $\sigma_{1} \sigma_{2}$-open and the sets in $\{\Phi,\{\mathrm{b}, \mathrm{c}\},\{\mathrm{c}\}, \mathrm{Y}\}$ are $\sigma_{1} \sigma_{2}$-closed. Let $\mathrm{f}: \mathrm{X} \rightarrow \mathrm{Y}$ be an identity mapping.Then $\mathrm{f}$ is $(1,2)^{*}-\pi \mathrm{gb}$ - continuous map but not $(1$, $2)^{*}$-continuous since $\mathrm{f}^{-1}(\mathrm{a}, \mathrm{b})=\{\mathrm{a}, \mathrm{b}\}$ which is not $\tau_{1} \tau_{2}$-open.

Composition of two $(1,2)^{*}-\pi \mathrm{gb}-$ continuous functions need not be $(1,2)^{*}-\pi \mathrm{gb}$-continuous.

Example 5.7: Let $X=\mathrm{Y}=\mathrm{Z},\{\mathrm{a}, \mathrm{b}, \mathrm{c}, \mathrm{d}\}, \tau_{1}=\{\Phi,\{\mathrm{b}\}$, $\{c\},\{b, c\}, X\}, \tau_{2}=\{\Phi, X\}$, So the sets in $\{\Phi,\{b\},\{c\},\{b, c\}, X\}$ are $\tau_{1} \tau_{2}$-open and the sets in $\{\Phi,\{a, c\},\{a, b\},\{a\}, X\}$ are $\tau_{1} \tau_{2}-$ closed. Let $\sigma_{1}=\{\Phi,\{a, b, d\}, Y\}$ and $\sigma_{2}=\{\Phi, Y\}$. So the sets in $\{\Phi,\{\mathrm{a}, \mathrm{b}, \mathrm{d}\}, \mathrm{Y}\}$ are $\sigma_{1} \sigma_{2}$-open and the sets in $\{\Phi,\{\mathrm{c}\}, \mathrm{Y}\}$ are $\sigma_{1} \sigma_{2}$-closed.Let $\eta_{1}=\{\Phi,\{a, d\}, X\}$ and $\eta_{2}=\{\Phi, Z\}$.So $\eta_{1} \eta_{2}-$ open $=\{\Phi,\{a, d\}, X\}$ and $\eta_{1} \eta_{2}$-closed $=\{\Phi,\{b, c\}, X\}$. Let f: $(X$, $\left.\tau_{1}, \tau_{2}\right) \rightarrow\left(\mathrm{Y}, \sigma_{1}, \sigma_{2}\right)$ by $\mathrm{f}(\mathrm{a})=\mathrm{a}, \mathrm{f}(\mathrm{b})=\mathrm{c}, \mathrm{f}(\mathrm{c})=\mathrm{b} . \mathrm{f}(\mathrm{d})=\mathrm{d}$. Define $\mathrm{g}:$ $\left(\mathrm{Y}, \sigma_{1}, \sigma_{2}\right) \rightarrow\left(\mathrm{Z}, \eta_{1}, \eta_{2}\right)$ by $\mathrm{g}(\mathrm{a})=\mathrm{d}, \mathrm{g}(\mathrm{b})=\mathrm{c}, \mathrm{g}(\mathrm{c})=\mathrm{b}, \mathrm{g}(\mathrm{d})=\mathrm{a}$. Then $f$ and $g$ are $(1,2)^{*}-\pi \mathrm{gb}$-continuous but gof is not $(1,2)^{*}$ $\pi \mathrm{gb}$-continuous since $\{\mathrm{b}, \mathrm{c}\}$ is $\eta_{1} \eta_{2}$-closed in $(Z, \eta)$ but (gof) ${ }^{1}(\{b, c\})=f^{-1}\left(g^{-1}(\{b, c\})=\{b, c\}\right.$ which is not $(1,2)^{*}-\pi$ gb-closed in $\left(\mathrm{X}, \tau_{1}, \tau_{2}\right)$.Hence gof is not $(1,2)^{*}-\pi \mathrm{gb}$-continuous.

Definition 5.8: A function f: $\left(\mathrm{X}, \tau_{1}, \tau_{2}\right) \rightarrow\left(\mathrm{Y}, \sigma_{1}, \sigma_{2}\right)$ is $\tau_{1} \tau_{2}-\pi$-open map if $\mathrm{f}(\mathrm{F})$ is $\tau_{1} \tau_{2}-\pi$-open map in $\mathrm{Y}$ for every $\tau_{1} \tau_{2}-\pi$-open in X.

Definition 5.9: A function $\mathrm{f}:\left(\mathrm{X}, \tau_{1}, \tau_{2}\right) \rightarrow\left(\mathrm{Y}, \sigma_{1}, \sigma_{2}\right)$ is $(1,2)^{*}$ - b-irresolute if for each b-open set $\mathrm{V}$ in $\mathrm{Y}, \mathrm{f}^{-1}(\mathrm{~V})$ is $\mathrm{b}$ open in X.

Theorem 5.10: If the bijective $\mathrm{f}:\left(\mathrm{X}, \tau_{1}, \tau_{2}\right) \rightarrow\left(\mathrm{Y}, \sigma_{1}, \sigma_{2}\right)$ is $(1,2) *$-b-irresolute and $\tau_{1} \tau_{2}-\pi$-open map ,then $\mathrm{f}$ is $(1,2)^{*}$ $\pi \mathrm{gb}-$ irresolute.

Proof: Let V be $(1,2)^{*}-\pi$ gb-closed in Y.Let $\mathrm{f}^{-1}(\mathrm{~V}) \subset \mathrm{U}$ where $U$ is $\tau_{1} \tau_{2}-\pi$-open in $X$. Then $V \subset f(U)$ where $f(U)$ is

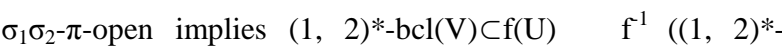
$(\mathrm{bcl}(\mathrm{V}))) \in \mathrm{U}$. Since $\mathrm{f}$ is $(1,2)^{*}$-b-irresolute, $\mathrm{f}^{-1}\left((1,2)^{*}\right.$ $(\mathrm{bcl}(\mathrm{V})))$ is $(1,2)^{*}$-b-closed in $\left(\mathrm{X}, \tau_{1}, \tau_{2}\right)$. Hence $(1,2)^{*}$-bcl(f $\left.{ }^{1}(\mathrm{~V})\right) \subset(1,2) *-b c l\left(f^{1}\left((1,2)^{*}-(\operatorname{bcl}(\mathrm{V}))\right)=\mathrm{f}^{-1}\left((1,2)^{*}\right.\right.$ $(\operatorname{bcl}(\mathrm{V}))) \subset \mathrm{U}$. Hence $\mathrm{f}^{-1}(\mathrm{~V})$ is $(1,2)^{*}-\pi \mathrm{gb}$-closed in $\left(\mathrm{X}, \tau_{1}, \tau_{2}\right)$. Therefore, $\mathrm{f}$ is $(1,2)^{*}-\pi \mathrm{gb}$ - irresolute.

Theorem 5.11: For any irresolute $(1,2) *$-irresolute map $\mathrm{f}: \mathrm{X} \rightarrow \mathrm{Y}$ and any $(1,2)^{*}-\pi \mathrm{gb}$-continuous map $\mathrm{g}: \mathrm{Y} \rightarrow \mathrm{Z}$, the composition $\mathrm{g} \circ \mathrm{f}: \mathrm{X} \rightarrow \mathrm{Z}$ is $(1,2)^{*}$ - $\pi \mathrm{gb}$-continuous map.

Proof: Let $V$ be $\eta_{1} \eta_{2}$-closed set in $Z$. Since $g: Y \rightarrow Z$ is $(1$, $2)^{*}-\pi \mathrm{gb}$-continuous map, $\mathrm{g}^{-1}(\mathrm{~V})$ is $(1,2)^{*}-\pi \mathrm{gb}$-closed in $\mathrm{Y}$.Since $\mathrm{f}: \mathrm{X} \rightarrow \mathrm{Y}$ is $(1,2)^{*}-\pi \mathrm{gb}$-irresolute map, $\mathrm{f}^{1}\left(\mathrm{~g}^{-1}(\mathrm{~V})\right)=(\mathrm{g} \circ \mathrm{f})^{-}$ ${ }^{1}(\mathrm{~V})$ is $(1,2)^{*}-\pi \mathrm{gb}$-closed in $\mathrm{X}$.Hence gof: $\mathrm{X} \rightarrow \mathrm{Z}$ is $(1,2)^{*}$ $\pi \mathrm{gb}$-continuous map.

From this paper we conclude the following results.
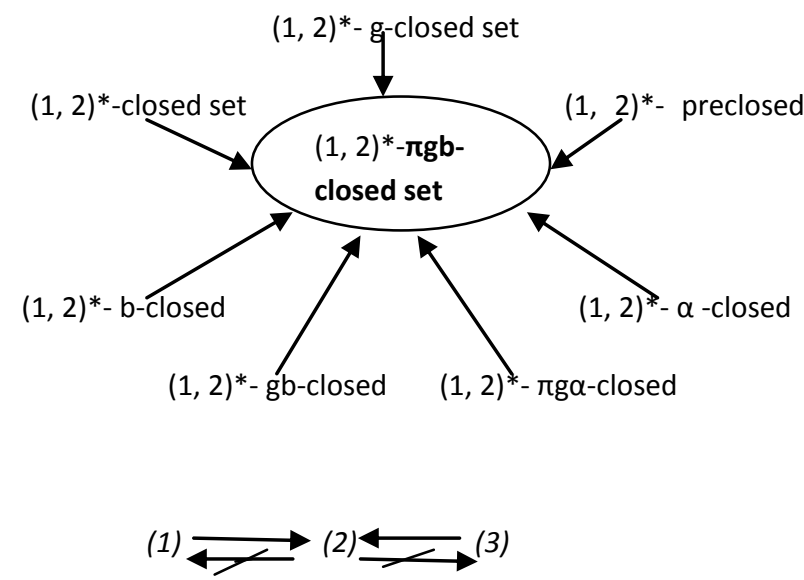

Where $(1)=(1,2) *$-continuity, $(2)=(1,2) *-\pi$ gb-continuity and $(3)=(1,2)^{*}-\pi \mathrm{gb}$-irresolute. 


\section{CONCLUSION}

This paper has attempted to compare $(1,2)^{*}-\pi$ gb-closed sets with the other closed sets in bitopological spaces.It also aims to state that the several definitions and results in this paper will result in obtaining several characterizations and also enable to study various properties. It brings to limelight that the weaker form of continuity in bitopological settings is the future scope of study.

\section{ACKNOWLEDGEMENT}

The author is delighted to express gratitude to the referees for their valuable comments and suggestions for the improvement of the paper.

\section{REFERENCES}

[1] D. Andrijevic, Semipreopen sets, Mat. Vesnik 8 (1986), no. 1, 24-32.

[2] S.P.Arya and T. M. Nour, Characterizations of s- normal spaces, Indian J. Pure Appl. Math.21 (1990) no 8, 717719.

[3] D. Andrijevic, On b-open sets, Mat. Vesnik48 (1996),no. 1-2, 59-64.

[4] G. Aslim1, A. Caksu Guler1 and T. Noiri, On $\pi g s^{-}$ closed sets in topological spaces Acta Math. Hungar.,) (2006), 275-283.

[5] Arockiarani and K. Mohana. $(1,2) *-\pi g \alpha$-closed sets and $(1,2)^{*}$ - quasi- $\alpha$ - normal spaces in bitopological settings. Antartica J. Math., 7(3), 45-355, 2010.

[6] M. Caldas and S. Jafari, On some applications of b-open sets in topological spaces, Kochi J.Math.2 (2007), 11-19.

[7] J.Dontchev, On generalizing semi-preopen sets,,Mem Fac Sci. Kochi Univ. Ser. A Math. 16 (1995), 35-48.

[8] J.Dontchev and M.Przemski,On the various decompositions of continuous and some weakly continuous functions, Acta Math. Hungar. 71 (1996), no.1-2, 109-120.

[9] E. Ekici and M. Caldas, Slightly -continuous functions, Bol. Soc. Parana. Mat. (3) 22 (2004),no. 2, 63-74.

[10] M. Ganster and M. Steiner, On some questions about bopen sets, Questions Answers Gen.Topology 25 (2007), no. 1, 45-52.

[11] C.Janaki, Studies on $\pi g \alpha$ - closed sets in Topology, Ph.D Thesis,Bharathiar University 2009.
[12] N. Levine, Semi-open sets and semi-continuity in topological spaces, Amer. Math. Monthly 70 (1963), 3641 .

[13] N. Levine, Generalized closed sets in topology ,Rend. Circ. Mat. Palermo (2) 1 (1970),89-96.

[14] M.Lellis Thivagar, B.MeeraDevi, Bitopological B-Open sets, International Journal of Algorithms, Computing and Mathematics, Volume 3, Number 3, August 2010

[15] S. Mashhour, M. E. Abd El-Monsef and S. N. El-Deep, On precontinuous and weak pre-Continuous mappings, Proc. Math. Phys. Soc. Egypt No. 53 (1982), 47-53 (1983).

[16] H. Maki, R. Devi and K. Balachandran, Associated topologies of generalized $\alpha$-closed sets and $\alpha$ generalized closed sets, Mem. Fac. Sci. Kochi Univ. Ser. A Math. (1994), 51-63.

[17] H.Maki, J.Umehara and T.Noiri,Every topological space is pre- $\mathrm{T}_{1 / 2}$, Mem. Fac. Sci. Kochi Univ. Ser. A Math. (1996), 33-4

[18] O. Njastad, On some classes of nearly open sets, Paci_c J. Math. (1965), 961-970.

[19] A. A. Nasef, On b-locally closed sets and related topics, Chaos Solitons Fractals (2001), no. 10, 1909-1915.

[20] A. Nasef, Some properties of contra--continuous functions, Chaos Solitons Fractals (2005), no. 2, 471477.

[21] J. H. Park, Strongly $\theta$-b-continuous functions,Acta Math.Hungar. (2006), no. 4, 347- 359.

[22] O.Ravi and M.Lellis Thivagar, On Stronger forms of $(1,2)^{*}$ - quotient mappings in bitopological spaces, Internat. J. Math. Game by and Algebra, 14(2004),no.6,481 -492.

[23] Saeid Jafari, M.Lellis Thivagar and Nirmala , On (1, 2) ${ }_{-\alpha} \hat{g}$ closed sets,J.Adv. Math.Studies Vol. 2(2009), No.2,25-34

[24] D. Sreeja and C. Janaki ,On $\pi$ gb- Closed Sets in Topological Spaces, International Journal of Mathematical Archive-2(8), 2011, 1314-1320. 